\title{
Optimum Plant Population of Bt and Non-Bt Corn in Wisconsin
}

\author{
Trenton F. Stanger* and Joseph G. Lauer
}

\begin{abstract}
Bt (Bacillus thuringiensis) corn (Zea mays L.) hybrids resist European corn borer [Ostrinia nubilalis (Hübner)] damage and lodge less, creating interest among growers, agronomists, and seed companies in their yield response to increasing plant population. Corn hybrids with $B t$ and non-Bt traits were evaluated from 2002 to 2004 across 10 locations in Wisconsin in 76-cm rows at target populations from 61750 to 123500 plants $\mathrm{ha}^{-1}$ to (i) determine the agronomic and economic optimum plant population for corn and (ii) identify agronomic and economic optimum plant populations for Bt and non-Bt hybrids. The quadratic model for both grain yield and grower return response to plant population was significant. The maximum yield plant population (MYPP) for Bt and non-Bt corn was 104500 and 98800 plants $\mathrm{ha}^{-1}$, respectively. The overall MYPP for corn was 102400 plants $\mathrm{ha}^{-1}$, which is 28300 plants $\mathrm{ha}^{-1}$ more than the current Wisconsin recommendation of 74100 plants ha $^{-1}$. Planting corn to the MYPP increased grain yield by $4.2 \%$ over the current population recommendation. However, the economically optimum plant population (EOPP) for both Bt and non-Bt corn was 83800 plants ha $^{-1}$. It was concluded that Bt corn hybrids require higher plant populations for maximizing yield potential; however the higher harvest costs related to those greater yields and the higher seed costs associated with attaining those populations resulted in no difference in the EOPP between Bt and non-Bt corn. Plant population recommendations for corn should be near 83800 plants ha ${ }^{-1}$, the point where the EOPP was achieved. Since this recommendation is affected by rising seed and management costs and variable market prices, a periodic evaluation of plant population response for newly released hybrids should be done.
\end{abstract}

$\mathrm{S}$ INCE 1996, several seed companies have commercialized new transgenic maize hybrids resistant to European Corn Borer (ECB) (Seydou et al., 2000). These new hybrids, commonly known as Bt corn, have been genetically engineered to incorporate genes of Bt (Koziel et al., 1993; Armstrong et al., 1995), a toxin effective against larvae from both first and second ECB generations. Resistance to second generation $\mathrm{ECB}$ will reduce stalk lodging, which may result in higher MYPP in Bt corn.

Lodging is a major constraint to maximizing grain yields in modern maize production (Sibale et al., 1992). High incidence of lodging is one of the hazards of increasing plant populations to get maximum yields. The most serious effect of denser stands is the higher incidence of stalk breakage (Stringfield and Thatcher, 1947). Crosbie (1982) showed that a large proportion of the grain yield improvement could be attributed to reduced stem lodging and ear droppage, and that the reduction in nonharvestable grain yield was most significant at higher

Department of Agronomy, 1575 Linden Dr., Univ. of Wisconsin, Madison, WI 53706. Funded by CSREES project WIS0 142-4897. Received 13 May 2005. *Corresponding author (tfstanger@wisc.edu).

Published in Agron. J. 98:914-921 (2006).

Corn

doi:10.2134/agronj2005.0144

(c) American Society of Agronomy

677 S. Segoe Rd., Madison, WI 53711 USA plant populations. Lodging can increase several-fold with high populations and may result in very high harvest losses that more than negate any yield increase that may have occurred with the higher plant population (Olson and Sander, 1988). The potential for increased lodging at high plant populations is a deterrent for recommending harvest populations for grain corn much above 74100 plants ha $^{-1}$ (Cox, 1997).

Lauer and Wedberg (1999) compared initial Bt corn hybrid introductions to their isolines, (the non-Bt equivalents of the Bt hybrids used) and to standard adapted hybrids in the northern U.S. Corn Belt. They concluded that yield of initial Bt hybrids was either equivalent to or better than standard adapted hybrids, except in environments with low incidence of ECB. Under low or no incidence of ECB initial Bt hybrids yielded less than standard adapted hybrids. Yields of isoline hybrids were $10 \%$ less than Bt hybrids and standard adapted hybrids, regardless of whether the ECB treatment was the infestation level, natural level, or ECB free. Stalk lodging was also greater in the high yielding standard hybrids compared to the Bt hybrids (7.1 vs. $2.5 \%$, respectively).

Graeber et al. (1999) compared Bt hybrids to non-Bt hybrids and concluded that the Bt hybrids reduced or eliminated first and second generation damage caused by ECB, yielded 4 to $6.6 \%$ greater than non-Bt hybrids, had decreased stalk lodging and greater test weight. The $\mathrm{Bt}$ and non-Bt hybrids were not different for tasseling date, silking date, leaf appearance date, individual leaf area, and plant height. Compared with non-Bt plants, $\mathrm{Bt}$ plants exhibited $9.7 \%$ greater total plant weight in 1997 and $9.4 \%$ greater grain yield in 1998 (Seydou et al., 2000).

According to Singer et al. (2003), the MYPP for Bt corn should be higher than non-Bt corn subjected to ECB damage because of the reduced potential for stalk lodging. Regression analysis of grain yield response to plant population for Bt and near-isoline non-Bt hybrids did not reveal a consistent hybrid response, although some evidence suggested Bt hybrids were more efficient than near-isolines at producing yield as plant population increased. The inability to identify different MYPPs for Bt and near-isolines was due to minimal stalk lodging and plant population treatments that did not maximize yield in most instances.

In a study conducted in New York during the 1970s, Knapp and Reid (1981) reported highest grain yield was attained at 54340 plants ha ${ }^{-1}$. Almost two decades later, Cox (1997) reported that MYPPs of individual hybrids ranged from 79040 to greater than 88920 plants ha $^{-1}$ under favorable conditions and from about 69160 to greater than 88920 plants ha $^{-1}$ under dry conditions. Frequency of drought is a concern that might deter a

Abbreviations: Bt, Bacillus thuringenisis; ECB, European corn borer; EOPP, economically optimum plant population; MN RM, Minnesota relative maturity [rating]; MYPP, maximum yield plant population. 
producer from increasing plant population. Cox (1997) stated that high plant populations do not decrease grain yields in dry years. In fact, plant population less than 74100 plants $\mathrm{ha}^{-1}$ resulted in more yield loss than observed for plant populations greater than 74100 plants $\mathrm{ha}^{-1}$, even under dry conditions.

In a population study conducted by Nafziger (1994), that had limited yields caused by drought, the MYPP was near 61750 plants $\mathrm{ha}^{-1}$, indicating that the risk of maintaining high populations even under dry conditions is not as high as it might have been with older hybrids. Newer hybrids appear to have improved ability to resist barrenness and other types of injury associated with high plant populations. According to Olson and Sander (1988), high plant populations are more water-use efficient than low plant populations. While water use is increased as plant population is increased, the increase is small and not proportional to stand increase.

Consequently, there appears to be minimum risk associated with planting high populations in a dry year other than additional seed costs. Carmer and Jackobs (1965) stated that reductions in yield are less rapid for populations in excess of the MYPP than for those below the MYPP. Such research demonstrates that it is a bigger risk not planting to the environment's maximum potential.

Cox (1997) suggested that periodic evaluations of plant population responses should be conducted on newly released hybrids in specific growing regions to accurately adjust plant population recommendations. Performance of new $\mathrm{Bt}$ hybrids have never been reported in a high stress, high population environment. The objectives of this study were (i) to determine the MYPP and EOPP for corn in Wisconsin and (ii) identify MYPP and EOPP for Bt and non-Bt hybrids.

\section{MATERIALS AND METHODS}

Experiments were conducted from 2002 to 2004 at 10 locations in Wisconsin. Trial locations were chosen that represented the diverse soils and climates of the state of Wisconsin. The locations were grouped into three relative maturity production zones. Soil characteristics at each location are listed in Table 1. All locations were managed according to recommended commercial production practices for maximizing grain yield. Cultural practices specific to each location are listed in Table 2 . Weeds were controlled by appropriate her- bicide treatments. To control corn rootworm (Diabrotica spp.), tefluthrin was applied in-furrow at a rate of $0.15 \mathrm{~kg}$ active ingredient (a.i.) ha ${ }^{-1}$ at planting to all locations that had corn as the preceding crop.

The experimental design at each location was a complete factorial arrangement of treatments in a randomized complete block with three replications. Factors were hybrid and plant population. Corn hybrids ranged from full-season to shorterseason maturity for their respective zones and were classified using the Minnesota relative maturity rating (Table 3). During 2002 , target plant populations of $61750,74100,86450,98800$, 111150 , and 123500 plants ha ${ }^{-1}$ were used and during 2003 and 2004 target plant populations of 64220, 79040, 93860, 108680 , and 123500 plants ha $^{-1}$ were used. The target plant populations used represented increments around the recommended plant population of 74100 plants ha $^{-1}$ and provided a range of plant populations for the covariate analysis.

Plots consisted of four rows (Arlington had eight rows) $6.7 \mathrm{~m}$ long and $0.76 \mathrm{~m}$ apart. Plots were over-planted and handthinned at growth stage V5-6 to achieve the desired target population (Ritchie et al., 1993). Plots were planted with a Kinze corn planter mounted with fluted cone units (Kinze Manufacturing, Williamsburg, IA).

Data collected before grain harvest included: preharvest plant population, ear population, dropped ears, and lodged plants. Plants were considered stalk lodged if corn stalks were broken below the ear or root lodged if the plants were leaning at greater than a $45^{\circ}$ angle. Percent lodging was calculated based on the total number of plants lodged plot $^{-1}$ divided by the total number of plants plot ${ }^{-1}$.

The two middle rows of each plot were harvested for grain yield with a self-propelled Kincaid Plot Combine (Kincaid Equipment Manufacturing, Haven, KS). The combine was equipped with a HarvestMaster GrainGage HM-1000 (Juniper Systems, Logan, UT) to measure plot grain yield, test weight, and moisture content. Plot yields were adjusted to a standard moisture content of $155 \mathrm{~g} \mathrm{~kg}^{-1}$.

In the economic analysis, grower return was determined using a partial budget that focused only on those costs and revenues that changed when plant population changed (Swinton and Lowenberg-DeBoer, 1998). There was no attempt to quantify costs associated with harvesting lodged corn. Grower return was the product of commodity price with yield subtracting production costs (Table 4). Costs were obtained from the "Estimated Costs of Crop Production in Iowa-2004 (Duffy, 2004) and Landmark Co-op (Cottage Grove, WI). Corn price was determined using a marketing strategy that had $50 \%$ of the crop sold in November and $25 \%$ forward contracted (less basis) to March and July, respectively. The November Average Cash price was derived from Wisconsin $\mathrm{Ag}$

Table 1. Soil type for 10 Wisconsin locations in 2002, 2003, and 2004.

\begin{tabular}{|c|c|c|}
\hline Location & Soil series name & Description and taxonomic name \\
\hline & & Southern zone \\
\hline $\begin{array}{l}\text { Arlington } \\
\text { Janesville } \\
\text { Lancaster }\end{array}$ & $\begin{array}{l}\text { Plano silt loam } \\
\text { Plano silt loam } \\
\text { Fayette silt loam }\end{array}$ & $\begin{array}{l}\text { fine-silty, mixed, superactive, mesic Typic Argiudolls } \\
\text { fine-silty, mixed, superactive, mesic Typic Argiudolls } \\
\text { fine-silty, mixed, superactive, mesic Typic Hapludalfs } \\
\text { South Central zone }\end{array}$ \\
\hline $\begin{array}{l}\text { Fond du Lac } \\
\text { Galesville } \\
\text { Hancock }\end{array}$ & $\begin{array}{l}\text { Virgil silt loam } \\
\text { Downs silt loam } \\
\text { Plainfield sand }\end{array}$ & $\begin{array}{l}\text { fine-silty, mixed, superactive, mesic Udollic Endoaqualfs } \\
\text { fine-silty, mixed, superactive, mesic Mollic Hapludalfs } \\
\text { mixed, mesic Typic Udipsamments } \\
\text { North Central zone }\end{array}$ \\
\hline $\begin{array}{l}\text { Chippewa Falls } \\
\text { Marshfield } \\
\text { Seymour } \\
\text { Valders }\end{array}$ & $\begin{array}{l}\text { Sattre silt loam } \\
\text { Loyal silt loam } \\
\text { Hortonville clay loam } \\
\text { Kewaunee clay loam }\end{array}$ & $\begin{array}{l}\text { fine-loamy over sandy or sandy-skeletal, mixed, superactive, mesic Mollic Hapludalfs } \\
\text { fine-loamy, mixed, superactive, frigid Oxyaquic Glossudalfs } \\
\text { fine-loamy, mixed, active, mesic Haplic Glossudalfs } \\
\text { fine, mixed, mesic Typic Hapludalfs }\end{array}$ \\
\hline
\end{tabular}


Table 2. Cultural practices for 10 Wisconsin locations in 2002, 2003, and 2004.

\begin{tabular}{|c|c|c|c|c|c|c|c|c|}
\hline \multirow[b]{2}{*}{ Location } & \multirow[b]{2}{*}{ Year } & \multirow[b]{2}{*}{ Plant date } & \multirow[b]{2}{*}{ Harvest date } & \multicolumn{5}{|c|}{ Soil test value } \\
\hline & & & & pH & Organic matter & $\mathbf{P}$ & $\mathbf{K}$ & Previous crop \\
\hline & & & & & $\%$ & $\longrightarrow$ & - & \\
\hline \multicolumn{9}{|c|}{ Southern zone } \\
\hline Arlington & 2002 & 30 Apr. & 21 Oct. & 6.2 & 3.3 & 79 & 247 & Soybean \\
\hline & 2003 & 3 May & 17 Oct. & 6.5 & 5.4 & 112 & 281 & Soybean \\
\hline & 2004 & 29 Apr. & 20 Oct. & 7.0 & 3.9 & 69 & 258 & Soybean \\
\hline Janesville & 2002 & 26 Apr. & 11 Oct. & 6.9 & 3.1 & 66 & 229 & Soybean \\
\hline & 2003 & 25 Apr. & 21 Oct. & 6.6 & 3.9 & 98 & 229 & Soybean \\
\hline & 2004 & 27 Apr. & 12 Oct. & 6.8 & 3.2 & 51 & 171 & Soybean \\
\hline Lancaster & 2002 & 26 Apr. & 14 Oct. & 7.3 & 2.1 & 57 & 157 & Soybean \\
\hline & 2003 & 28 Apr. & 7 Oct. & 6.9 & 2.3 & 53 & 80 & Soybean \\
\hline & 2004 & 27 Apr. & 14 Oct. & 7.4 & 2.1 & 60 & 147 & Soybean \\
\hline \multicolumn{9}{|c|}{$\underline{\text { South Central zone }}$} \\
\hline Fond du Lac & 2002 & 10 May & 17 Oct. & 7.1 & 4.2 & 42 & 100 & Soybean \\
\hline & 2003 & 3 May & 22 Oct. & 6.7 & 2.9 & 47 & 57 & Soybean \\
\hline Galesville & 2002 & 3 May & 9 Oct. & 6.6 & 3.2 & 43 & 161 & Soybean \\
\hline & 2003 & 28 Apr. & 20 Oct. & 6.5 & 3.1 & 47 & 85 & Soybean \\
\hline & 2004 & 28 Apr. & 25 Oct. & 6.1 & 3.8 & 22 & 150 & Soybean \\
\hline Hancock & 2002 & 29 Apr. & 10 Oct. & 6.4 & 0.7 & 120 & 47 & Soybean \\
\hline & 2003 & 24 Apr. & 15 Oct. & 6.2 & 0.8 & 112 & 30 & Soybean \\
\hline & 2004 & 24 Apr. & 13 Oct. & 6.8 & 0.7 & 98 & 96 & Soybean \\
\hline \multicolumn{9}{|c|}{ North Central zone } \\
\hline Chippewa Falls & 2002 & 3 May & 16 Oct. & 6.4 & 2.1 & 30 & 111 & Soybean \\
\hline & 2003 & 29 Apr. & 24 Oct. & 6.5 & 2.4 & 26 & 61 & Soybean \\
\hline & 2004 & 28 Apr. & 25 Oct. & 6.4 & 2.1 & 25 & 109 & Soybean \\
\hline \multirow[t]{3}{*}{ Marshfield } & 2002 & 15 May & 31 Oct. & 6.4 & 3.1 & 62 & 172 & Soybean \\
\hline & 2003 & 1 May & 8 Oct. & 6.5 & 3.4 & 66 & 109 & Alfalfa \\
\hline & 2004 & 29 Apr. & 3 Nov. & 6.5 & 2.9 & 38 & 103 & Soybean \\
\hline \multirow{2}{*}{ Seymour } & 2003 & 2 May & 24 Oct. & 7.3 & 2.8 & 25 & 97 & Soybean \\
\hline & 2004 & 2 May & 24 Oct. & 7.5 & 2.6 & 41 & 179 & Corn \\
\hline \multirow[t]{3}{*}{ Valders } & 2002 & 15 May & 17 Oct. & 6.4 & 3.1 & 62 & 172 & Corn \\
\hline & 2003 & 2 May & 24 Oct. & 6.8 & 3.6 & 102 & 110 & Corn \\
\hline & 2004 & 4 May & 27 Oct. & 6.9 & 4.1 & 91 & 186 & Corn \\
\hline
\end{tabular}

Statistics, and the March and July future prices were derived from the Chicago Board of Trade Futures price on the first business day in December.

Table 3. Corn hybrids tested at three Wisconsin production zones during 2002, 2003, and 2004.

\begin{tabular}{|c|c|c|c|}
\hline Year & Hybrid & Trait & $\mathbf{M N} \mathbf{R M}+$ \\
\hline \multicolumn{4}{|c|}{ Southern zone } \\
\hline 2002 & $\begin{array}{l}\text { Pioneer 37R71 } \\
\text { Dairyland } 1410\end{array}$ & $\begin{array}{l}\text { Bt } \\
\text { non-Bt }\end{array}$ & $\begin{array}{l}99 \mathrm{~d} \\
110 \mathrm{~d}\end{array}$ \\
\hline 2003 & Pioneer 37R71 & Bt & $99 \mathrm{~d}$ \\
\hline & Renk RK622 & non-Bt & $100 \mathrm{~d}$ \\
\hline & Pioneer 34M95 & & $109 d$ \\
\hline & Pioneer 34M94 & non-Bt & $109 \mathrm{~d}$ \\
\hline 2004 & Pioneer 34M95 & Bt & $109 d$ \\
\hline & Pioneer 34M94 & non-Bt & $109 d$ \\
\hline & Jung HDS104 & non-Bt & $106 \mathrm{~d}$ \\
\hline \multicolumn{4}{|c|}{$\underline{\text { South Central zone }}$} \\
\hline 2002 & Pioneer 37R71 & Bt & $99 d$ \\
\hline & Dekalb DKC4442 & Bt & $95 \mathrm{~d}$ \\
\hline 2003 & Pioneer 37R71 & Bt & $99 \mathrm{~d}$ \\
\hline & Renk RK622 & non-Bt & $100 \mathrm{~d}$ \\
\hline & Dekalb DK5018 & Bt & $100 \mathrm{~d}$ \\
\hline & Dekalb DK5143 & non-Bt & $100 \mathrm{~d}$ \\
\hline 2004 & Dekalb DK5018 & Bt & $100 \mathrm{~d}$ \\
\hline & Dekalb DK5143 & non-Bt & $100 \mathrm{~d}$ \\
\hline \multicolumn{4}{|c|}{ North Central zone } \\
\hline 2002 & Pioneer 37R71 & Bt & $99 \mathrm{~d}$ \\
\hline & Cargill 4521Bt & Bt & $105 d$ \\
\hline \multirow[t]{4}{*}{2003} & Pioneer 37R71 & & $99 \mathrm{~d}$ \\
\hline & Renk RK622 & non-Bt & $100 \mathrm{~d}$ \\
\hline & NK Brand N3030Bt & & $93 \mathrm{~d}$ \\
\hline & NK Brand N3030 & non-Bt & $95 d$ \\
\hline \multirow[t]{2}{*}{2004} & NK Brand N3030Bt & Bt & $93 \mathrm{~d}$ \\
\hline & NK Brand N3030 & non-Bt & $95 \mathrm{~d}$ \\
\hline
\end{tabular}

$\dagger$ Minnesota relative maturity rating.
Data were analyzed by covariate analysis using the PROC MIXED procedure (Littell et al., 1996) of SAS (SAS Institute, 2001). The covariate was plant population at harvest. Thus, the data from each individual plot was used and not the treatment means. Data were pooled over years by location, zone, and overall. For determining the expected mean squares and appropriate $F$ tests in the analysis of covariance, random effects for the location analysis were year, $\operatorname{rep}($ location $\times$ year), zone $\times$ year, and hybrid(trait); for the zone analysis random effects were year, location(year), rep(location $\times$ year), and hybrid (trait); and year, location(zone $\times$ year), rep $\times$ location(zone $\times$ year), and hybrid(trait) for the overall analysis. Least square means of the fixed effects were computed, and the PDIFF option of the LSMEANS statement was used to display the differences among least square means for comparison. This option uses Fisher's protected least significant difference, and comparison was conducted at $P \leq 0.05$. The coefficient of determination $\left(R^{2}\right)$ was derived using the predicted values calculated by PROC MIXED $\left(R^{2}=1-\left[\left(y_{\mathrm{ij}}-y_{(\text {Pred })}\right)^{2} /\left(y_{\mathrm{ij}}-\right.\right.\right.$ $\left.\left.y_{\text {(grand mean) }}\right)^{2}\right]$ ).

The final model that was used to determine each of the regression equations was attained using backward stepwise selection. This procedure starts with the full model and sequentially deletes factors and their interactions. The factor producing the smallest $F$ value is deleted at each stage and the model is complete when the factors remaining in the model produce a value of $P \leq 0.05$.

For each location-trait, zone-trait, and overall-trait data pool, the maximum yield, maximum grower return, and their respective plant populations were calculated. Maximum yield plant population and EOPP were calculated by taking the first derivative of either the yield or economic function and solving for $X$ (plant population). The plant population at $95 \%$ of maximum yield was also calculated to account for any upward bias 
Table 4. Economic values for determining grower return for 2002-2004.

\begin{tabular}{|c|c|c|c|c|}
\hline & 2002 & 2003 & 2004 & Avg. \\
\hline $\begin{array}{l}\text { Market price }\left(\$ \mathrm{Mg}^{-1}\right) \\
\text { Retail seed prices (\$ per } \\
80000 \text { seed bag) }\end{array}$ & 88.20 & 88.20 & 82.29 & 86.23 \\
\hline $\begin{array}{l}\text { Bt hybrids } \\
\text { Non-Bt hybrids }\end{array}$ & $\begin{array}{r}121.45 \\
99.90\end{array}$ & $\begin{array}{r}126.44 \\
99.74\end{array}$ & $\begin{array}{l}137.13 \\
108.60\end{array}$ & $\begin{array}{l}128.34 \\
102.75\end{array}$ \\
\hline \multicolumn{5}{|l|}{$\underline{\text { Harvest costs }}$} \\
\hline 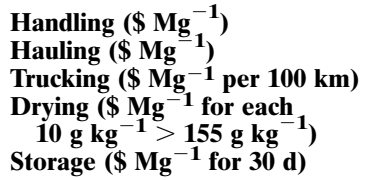 & $\begin{array}{l}0.63 \\
1.26 \\
7.88 \\
1.18 \\
1.34\end{array}$ & & & \\
\hline
\end{tabular}

created by our quadratic model. If the calculated optimum plant population was outside of the plant population range for each data pool combination, then the optimum plant population was the same as the population of the actual maximum yield or grower return. If the calculated plant population at 95\% maximum $Y$ was outside of the plant population treatments for each data pool combination, then the plant population at $95 \%$ of maximum $Y$ equaled the lowest actual plant population tested at that location (Lauer et al., 1999).

Maximum net returns to seed costs for the economic model were obtained by equating the first derivatives of the grower return response equation to zero, solving for $X$ (EOPP), substituting $X$ into the response equation and solving for maximum return. The $\pm \$ 2.50 \mathrm{ha}^{-1}$ error bars were calculated by taking the maximum net returns to seed costs subtracting $\$ 2.50$ and solving for $X$ using the quadratic equation. Seed cost/ market price ratio is calculated using seed costs as $\$ 1000$ seeds ${ }^{-1}$ and market price as $\$ \mathrm{Mg}^{-1}$.

\section{RESULTS AND DISCUSSION}

Growing conditions varied considerably among locations and years. In 2002, warm, dry conditions during April were followed by cool, wet conditions during early May to early June and followed by hot, dry conditions in early July. Hot and dry weather during pollination lowered yields in Fond du Lac, Janesville, Seymour, and Valders. Timely rains in Chippewa Falls, Galesville, and Lancaster resulted in favorable pollination and grain yields. In Seymour, cool, wet conditions during April and May severely affected corn emergence, development, and subsequent stand establishment causing this location to be dropped from the analysis for 2002 .

In 2003, spring conditions were cooler and dryer than average, followed by hot, dry conditions in mid-July. Favorable plant emergence resulted in adequate stand establishment at most locations. However, emerged stands at Marshfield and Valders were poor due to a cold, wet spring at Marshfield and heavy crusting at Valders, but plant population treatments were able to be applied within target levels at both locations. Chippewa Falls and Lancaster had reduced grain yields caused by dry, hot weather from mid-July through August. Although variable, grain yields were above average at Seymour and Valders.

In 2004, spring conditions were good through early May followed by cooler and wetter than average conditions. Record rainfall occurred during May and early
June at Chippewa Falls, Fond du Lac, Marshfield, Seymour, and Valders. Cool conditions slowed early season development, however good growing conditions during the summer allowed crop development to catch up during September. Grain yields were above average at Lancaster. At Fond du Lac, wet field conditions after planting severely affected corn emergence, development, and subsequent stand establishment causing this location to be dropped from the analysis for 2004.

\section{Grain Yield}

The significance of $F$ values attained from the analyses of covariance for grain yield, grain moisture, lodging, and grower return are shown in Table 5. The covariate variable (harvest plant population) was significant for grain yield for all three data pools (Table 5). Increasing plant population significantly increased grain yield (Table 6 and Fig. 1). A significant plant population $\times$ trait interaction for grain yield was observed for all three data pools (Table 5). As harvest plant population increased from 64220 to 123500 plants ha ${ }^{-1}$, Bt corn yielded 5.9 to $8.0 \%$ more than non-Bt corn, respectively (Table 6 and Fig. 1). This yield increase for Bt hybrids was consistent with Bode and Calvin (1990), Graeber et al. (1999), Lauer and Wedberg (1999), and Dillehay et al. (2004).

Table 5. Significance of $F$ values from analysis of covariance of grain yield, grain moisture, lodging, and grower return, 2002 to 2004. Data were pooled over years by location, zone, and overall.

\begin{tabular}{|c|c|c|c|c|}
\hline Source of variation & $\begin{array}{l}\text { Grain } \\
\text { yield }\end{array}$ & $\begin{array}{c}\text { Grain } \\
\text { moisture }\end{array}$ & Lodging & $\begin{array}{l}\text { Grower } \\
\text { return }\end{array}$ \\
\hline \multicolumn{5}{|l|}{$\underline{\text { Location }}$} \\
\hline Trait (T) & $\mathbf{N S} \dagger$ & NS & NS & NS \\
\hline Location (L) & $*$ & **** & ** & $* *$ \\
\hline $\mathbf{T} \times \mathbf{L}$ & NS & $*$ & $*$ & NS \\
\hline $\begin{array}{l}\text { Harvest plant } \\
\text { population (P) }\end{array}$ & $* * *$ & NS & NS & $* * *$ \\
\hline $\mathbf{P} \times \mathbf{T}$ & ** & NS & NS & NS \\
\hline $\mathbf{P} \times \mathbf{L}$ & $* * *$ & $* * *$ & $* * *$ & $* * *$ \\
\hline $\mathbf{P} \times \mathbf{T} \times \mathbf{L}$ & $* *$ & $*$ & **** & $*$ \\
\hline $\mathbf{P}^{2}$ & $* * *$ & NS & **** & $* * *$ \\
\hline $\mathbf{P}^{2} \times \mathbf{T}$ & NS & NS & NS & NS \\
\hline $\mathbf{P}^{2} \times \mathbf{L}$ & $* * *$ & *** & NS & $* *$ \\
\hline $\mathbf{P}^{2} \times \mathbf{T} \times \mathbf{L}$ & NS & $*$ & NS & NS \\
\hline \multicolumn{5}{|l|}{ Zone } \\
\hline $\mathbf{T}$ & NS & NS & NS & NS \\
\hline Zone (Z) & ** & $*$ & NS & $* * * *$ \\
\hline $\mathbf{T} \times \mathbf{Z}$ & NS & $* *$ & $* *$ & NS \\
\hline $\mathbf{P}$ & $* * *$ & $* * *$ & NS & $* * *$ \\
\hline $\mathbf{P} \times \mathbf{T}$ & * & NS & NS & NS \\
\hline $\mathbf{P} \times \mathbf{Z}$ & NS & *** & $* * *$ & NS \\
\hline $\mathbf{P} \times \mathbf{T} \times \mathbf{Z}$ & NS & NS & $*$ & NS \\
\hline $\mathbf{P}^{2}$ & $* * *$ & NS & $* *$ & $* * * *$ \\
\hline $\mathbf{P}_{2}^{2} \times \mathbf{T}$ & NS & NS & NS & NS \\
\hline $\mathbf{P}_{2}^{2} \times \mathbf{Z}$ & $* *$ & NS & NS & * \\
\hline $\mathbf{P}^{2} \times \mathbf{T} \times \mathbf{Z}$ & NS & NS & NS & NS \\
\hline \multicolumn{5}{|l|}{ Overall } \\
\hline $\mathbf{T}$ & NS & NS & NS & NS \\
\hline $\mathbf{P}$ & $* * *$ & $* * *$ & $*$ & $* * *$ \\
\hline $\mathbf{P} \times \mathbf{T}$ & $*$ & NS & $*$ & NS \\
\hline $\mathbf{P}_{2}^{2}$ & $* * *$ & NS & $* * *$ & $* * *$ \\
\hline $\mathbf{P}^{2} \times \mathbf{T}$ & NS & NS & NS & NS \\
\hline
\end{tabular}

* Significant at the 0.05 level.

** Significant at the 0.01 level.

*** Significant at the 0.001 level.

$\dagger \mathbf{N S}$, no significant differences at $P \leq \mathbf{0 . 0 5}$. 
Table 6. Regression equations and predicted corn yields for selected plant populations, 2002 to 2004.

\begin{tabular}{|c|c|c|c|c|c|c|c|c|c|c|c|c|c|c|}
\hline \multirow[b]{3}{*}{ Data pool } & \multirow[b]{3}{*}{ Trait } & \multirow[b]{3}{*}{$\mathbf{L S D} \dagger$} & \multirow{2}{*}{\multicolumn{3}{|c|}{ Polynomial regression $₹$}} & \multirow[b]{3}{*}{$R^{2} \S$} & & & edicted & ield & & \multirow{3}{*}{$\begin{array}{c}\text { Maximum } \\
\text { yield }\end{array}$} & \multirow{3}{*}{$\begin{array}{c}\text { Population } \\
\text { at } 95 \% \text { of } \\
\text { maximum yield }\end{array}$} & \multirow{3}{*}{$\begin{array}{l}\text { Maximum } \\
\text { yield plant } \\
\text { population }\end{array}$} \\
\hline & & & & & & & \multicolumn{5}{|c|}{ Final population (plants ha ${ }^{-1}$ ) } & & & \\
\hline & & & \multicolumn{3}{|c|}{$Y=A+B(P)+C\left(P^{2}\right)$} & & 64220 & 79040 & 93860 & 108680 & 123500 & & & \\
\hline \multicolumn{3}{|l|}{ Location } & $\mathbf{A}$ & B & $\mathbf{C}$ & & \multicolumn{6}{|c|}{$-\mathrm{Mg} \mathrm{ha}^{-1}$} & \multicolumn{2}{|c|}{ plants ha ${ }^{-1}$} \\
\hline Arlington & Bt & c-g & 3.89 & 1.75 & -0.087 & 0.52 & 11.6 & 12.3 & 12.7 & 12.7 & 12.3 & 12.7 & 73700 & 100800 \\
\hline Arlington & non-Bt & d-i & 5.85 & 1.46 & -0.087 & 0.56 & 11.6 & 11.9 & 11.9 & 11.4 & 10.6 & 12.0 & 57600 & 83800 \\
\hline Chippewa Falls & Bt & hj & 7.98 & 0.51 & -0.028 & 0.78 & 10.1 & 10.3 & 10.4 & 10.3 & 10.1 & 10.4 & 59700 & 92800 \\
\hline Chippewa Falls & non-Bt & $\mathbf{k}$ & 8.02 & 0.32 & -0.028 & 0.96 & 8.9 & 8.8 & 8.6 & 8.2 & 7.8 & 8.9 & 54800 & 57800 \\
\hline Fond du Lac & Bt & c-h & 8.94 & 0.63 & -0.029 & 0.63 & 11.8 & 12.2 & 12.4 & 12.4 & 12.4 & 12.5 & 64200 & 111000 \\
\hline Fond du Lac & non-Bt & e-j & 8.49 & 0.59 & -0.029 & 0.79 & 11.1 & 11.4 & 11.5 & 11.5 & 11.4 & 11.6 & 58700 & 103700 \\
\hline Galesville & Bt & bed & 4.54 & 1.79 & -0.084 & 0.87 & 12.5 & 13.4 & 13.9 & 14.0 & 13.8 & 14.0 & 77200 & 106100 \\
\hline Galesville & non-Bt & $e-i$ & 4.80 & 1.61 & -0.084 & 0.77 & 11.7 & 12.3 & 12.5 & 12.4 & 11.9 & 12.5 & 68600 & 95900 \\
\hline Hancock & Bt & a & 6.66 & 1.69 & -0.073 & 0.98 & 14.5 & 15.4 & 16.0 & 16.3 & 16.3 & 16.3 & 81400 & 114700 \\
\hline Hancock & non-Bt & ab & 6.38 & 1.61 & -0.073 & 0.98 & 13.7 & 14.5 & 15.1 & 15.2 & 15.1 & 15.2 & 77600 & 109800 \\
\hline Janesville & Bt & bce & 6.53 & 1.33 & -0.063 & 0.65 & 12.4 & 13.1 & 13.4 & 13.5 & 13.3 & 13.5 & 72200 & 104900 \\
\hline Janesville & non-Bt & bce & 5.26 & 1.45 & -0.063 & 0.65 & 12.0 & 12.8 & 13.3 & 13.6 & 13.5 & 13.6 & 81900 & 114600 \\
\hline Lancaster & Bt & b-f & 0.48 & 2.75 & -0.145 & 0.82 & 12.2 & 13.1 & 13.5 & 13.2 & 12.3 & 13.5 & 73100 & 94600 \\
\hline Lancaster & non-Bt & c-h & 1.56 & 2.55 & -0.145 & 0.86 & 11.9 & 12.6 & 12.7 & 12.1 & 10.9 & 12.7 & 66800 & 87700 \\
\hline Marshfield & Bt & gh & 6.96 & 0.69 & $-\mathbf{0 . 0 3 0}$ & 0.94 & 10.2 & 10.5 & 10.8 & 10.9 & 10.9 & 10.9 & 71700 & 114100 \\
\hline Marshfield & non-Bt & jk & 6.82 & 0.58 & $-\mathbf{0 . 0 3 0}$ & 0.98 & 9.3 & 9.5 & 9.6 & 9.5 & 9.3 & 9.6 & 55400 & 95100 \\
\hline Seymour & Bt & c-g & 3.85 & 1.55 & -0.070 & 0.95 & 10.9 & 11.7 & 12.2 & 12.4 & 12.3 & 12.5 & 81200 & 111000 \\
\hline Seymour & non-Bt & e-i & 3.02 & 1.56 & -0.070 & 0.94 & 10.1 & 11.0 & 11.5 & 11.7 & 11.6 & 11.7 & 82600 & 111600 \\
\hline Valders & Bt & e-i & 4.15 & 1.59 & -0.083 & 0.60 & 10.9 & 11.5 & 11.7 & 11.6 & 11.1 & 11.7 & 69100 & 95700 \\
\hline Valders & non-Bt & fgh & 2.41 & 1.72 & -0.083 & 0.73 & 10.0 & 10.8 & 11.2 & 11.3 & 10.9 & 11.3 & 77300 & 103400 \\
\hline \multicolumn{15}{|l|}{ Zone } \\
\hline NC & & b & 4.41 & 1.33 & -0.067 & 0.85 & 10.2 & 10.8 & 11.1 & 11.0 & 10.7 & 11.1 & 71400 & 100300 \\
\hline NC & non-Bt & b & 4.16 & 1.27 & -0.067 & 0.94 & 9.6 & 10.0 & 10.2 & 10.1 & 9.7 & 10.2 & 67700 & 95400 \\
\hline SC & Bt & a & 7.11 & 1.33 & -0.060 & 0.94 & 13.2 & 13.9 & 14.3 & 14.5 & 14.4 & 14.5 & 75900 & 110600 \\
\hline SC & non-Bt & a & 6.85 & 1.27 & -0.060 & 0.92 & 12.5 & 13.1 & 13.5 & 13.5 & 13.3 & 13.5 & 71700 & 105200 \\
\hline $\mathbf{S}$ & Bt & a & 6.55 & 1.33 & -0.066 & 0.71 & 12.4 & 13.0 & 13.2 & 13.2 & 12.9 & 13.3 & 69100 & 100800 \\
\hline $\mathbf{S}$ & non-Bt & $\mathbf{a}$ & 6.29 & 1.27 & -0.066 & 0.68 & 11.7 & 12.2 & 12.4 & 12.3 & 11.9 & 12.4 & 65300 & 95900 \\
\hline \multicolumn{15}{|l|}{ Overall } \\
\hline & & $\mathbf{a}$ & 6.11 & 1.27 & -0.061 & 0.86 & 11.8 & 12.4 & 12.7 & 12.7 & 12.5 & 12.8 & 72100 & 104500 \\
\hline & Bt & a & 5.90 & 1.20 & $-\mathbf{0 . 0 6 1}$ & 0.89 & 11.1 & 11.6 & 11.8 & 11.8 & 11.5 & 11.8 & 67600 & 98800 \\
\hline
\end{tabular}

$\dagger$ LSD, Least Significant Difference as calculated by Proc Mixed. Averaged across plant population treatments, grain yield means from areas and traits followed by the same letter are not significantly different $(P \leq 0.05)$.

$\ddagger \mathrm{Y}$, corn grain yield in $\mathrm{Mg} \mathrm{ha}^{-1} ; P=$ final plant population in plants ha ${ }^{-1} / 10000$.

$\S R^{2}$, coefficient of determination.

The analysis also showed the quadratic coefficient of plant population to be significant (Tables 5 and 6). In previous plant population studies, Nafziger (1994) and Porter et al. (1997) found that the quadratic model pro- vided the best fit. Using a quadratic model of the harvest plant population to fit the data, we observed high coefficients of determination $\left(R^{2}\right)$ in all data pools (Table 6). The quadratic coefficients of plant population were sig-

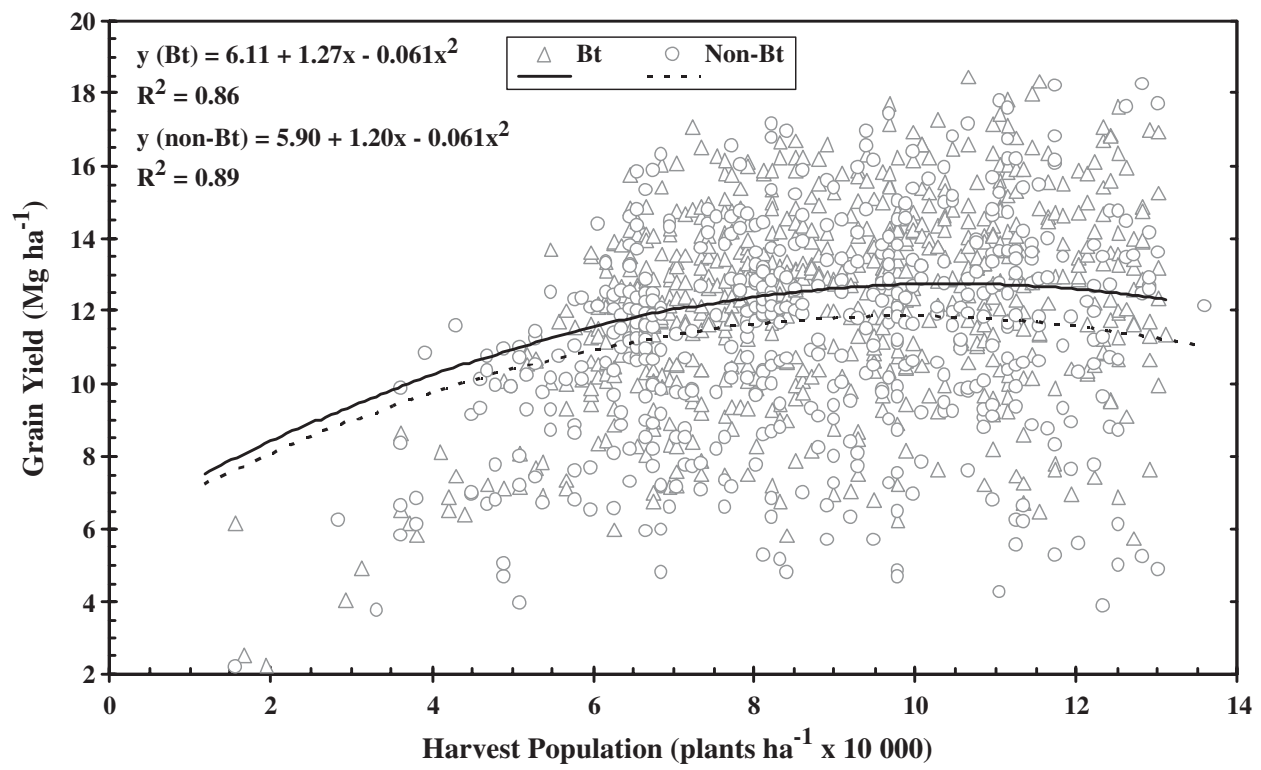

Fig. 1. The relationship between plant population and grain yield for Bt and non-Bt hybrids in Wisconsin during 2002 to 2004. Points represent individual plots. 
nificant and negative across all pooling groups (Tables 5 and 6), indicating that the plant population reached a point that was too high to represent the maximum attainable corn yield. The overall MYPP for Bt and nonBt corn was 104500 and 98800 plants ha $^{-1}$, respectively (Table 6 and Fig. 1). The overall MYPP was 102400 plants ha ${ }^{-1}$. This is 28300 plants ha $^{-1}$ more than the current recommendation. However, if growers use the current recommendation of 74100 plants $\mathrm{ha}^{-1}$, grain yield would be $95.6 \%(\mathrm{Bt})$ and $96.9 \%$ (non-Bt) of the grain yield at MYPP (Table 6).

Overall, when plant populations were increased from 74100 plants ha ${ }^{-1}$ to 102400 plants ha ${ }^{-1}$, yields increased by $4.2 \%$. Newer hybrids have improved stress tolerance associated with plant populations above what is currently recommended (Duvick and Cassman, 1999). However, not all environments were favorable to these high plant populations. For example, non-Bt hybrids at Chippewa Falls produced their highest yields at the lowest tested plant population (Table 6).

\section{Grain Moisture}

Overall, there was no difference in grain moisture at harvest between $\mathrm{Bt}$ and non-Bt corn. These results contradicted previous research (Dillehay et al., 2004; Bruns and Abbas, 2006) regarding grain moisture for Bt hybrids and their respective isolines. In those studies, an increase in grain moisture for Bt hybrids was observed. However, our study compared a different set of Bt and non-Bt hybrids (Bt, non-Bt equivalents, and standard adapted hybrids) than the previous literature. From a production standpoint, this moisture difference is minor and would not likely affect the price received for the grain on the market (Bruns and Abbas, 2006).

Harvest population affected grain moisture content (Table 5). Overall, grain moisture decreased $1.7 \%$ when harvest plant population increased from 64220 to 123500 plants $\mathrm{ha}^{-1}$. This observation was consistent with Widdicombe and Thelen (2002) where grain moisture decreased 5.2 and $3.5 \%$ with increasing plant populations for early and mid-season hybrids, respectively. Other than at high plant populations, a difference in grain moisture was seen sporadically and attributed to a variety of factors including weather, soil, location, and hybrid. Widdicombe and Thelen (2002) also observed differences in grain moisture among hybrids, which were the result of differences in relative maturity and the time they were allowed to field-dry before harvest. The relative maturities of the hybrids used in this study, although adapted to their zones, were not the same in all locations.

\section{Lodging}

Harvest plant population affected lodging. An increase in harvest plant population from 64220 to 123500 plants ha ${ }^{-1}$ increased lodging from 5.0 to $15.8 \%$. Pedersen and Lauer (2002) and Bruns and Abbas (2005) found similar results regarding higher plant population effects on lodging percentage. In addition, a significant plant population $\times$ trait (overall pool) interaction was observed (Table 5). As plant population increased, from 64220 to 123500 plants ha ${ }^{-1}$, non-Bt corn experienced $22 \%$ more lodging than Bt corn. The quadratic coefficient of plant population showed significance in all data pools (Table 5). As plant population increased, the rate of increase for lodging became greater. At low and moderate populations, lodging severity varied considerably depending on location and year. Some of this lodging variability was attributed to stalk rot that was more severe at some locations than others.

\section{Grower Return}

Harvest plant population had a significant effect on grower return for all data pools (Table 5). The covariate analysis indicated that the linear and quadratic coefficients of plant population were significant (Table 5 and 7). Grower return increased with increasing plant population to a point of maximum return and then declined (Table 7). Despite Bt corn yielding 6.6\% more than nonBt corn, the yield benefit was offset by the higher costs of seed, handling, hauling, trucking, and storage resulting in no economic benefit to Bt corn. For both Bt and non-Bt hybrids, grower return was optimized at a plant population of 83800 plants ha ${ }^{-1}$ (Table 7). This population is 9700 plants ha ${ }^{-1}$ greater than the currently recommended plant population of 74100 plants ha $^{-1}$.

These results were attained using actual seed costs and market prices effective at the time. Corn growers base their plant population decisions on yield response,

Table 7. Regression equations and predicted grower return for selected plant populations, 2002 to 2004.

\begin{tabular}{|c|c|c|c|c|c|c|c|c|c|c|c|c|}
\hline \multirow[b]{3}{*}{ Data pool } & \multirow[b]{3}{*}{$\mathbf{L S D} \dagger$} & \multirow{2}{*}{\multicolumn{3}{|c|}{ Polynomial regression $\ddagger$}} & \multicolumn{6}{|c|}{ Predicted return } & \multirow{3}{*}{$\begin{array}{c}\text { Maximum } \\
\text { grower return }\end{array}$} & \multirow{3}{*}{$\begin{array}{l}\text { Economic optimum } \\
\text { plant population }\end{array}$} \\
\hline & & & & & \multirow[b]{2}{*}{$R^{2} \S$} & \multicolumn{5}{|c|}{ Final population (plants ha ${ }^{-1}$ ) } & & \\
\hline & & \multicolumn{3}{|c|}{$Y=A+B(P)+C\left(P^{2}\right)$} & & 64220 & 79040 & 93860 & 108680 & 123500 & & \\
\hline Zone & & $\mathbf{A}$ & B & C & & & & 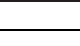 & $\mathrm{ha}^{-1}$ & & & plants ha ${ }^{-1}$ \\
\hline NC & b & 186 & 73.2 & -4.49 & 0.90 & 471 & 484 & 478 & 451 & 405 & 485 & 81500 \\
\hline SC & $\mathbf{a}$ & 404 & 73.2 & -4.14 & 0.92 & 703 & 724 & 726 & 710 & 676 & 727 & 88400 \\
\hline $\mathbf{S}$ & a & 383 & 73.2 & -4.40 & 0.75 & 672 & 687 & 683 & 659 & 616 & 688 & 83200 \\
\hline \multicolumn{13}{|l|}{ Overall } \\
\hline & - & 328 & 69.4 & -4.14 & 0.88 & 706 & 719 & 714 & 692 & 653 & 619 & 83800 \\
\hline
\end{tabular}

$\dagger$ LSD, Least Significant Difference as calculated by Proc Mixed. Averaged across plant population treatments, grower return means from areas followed by the same letter are not significantly different $(P \leq 0.05)$.

$\ddagger Y$, grower return in $\$ h^{-1} ; P=$ final plant population in plants $h^{-1} / 10000$.

$\S R^{2}$, coefficient of determination. 


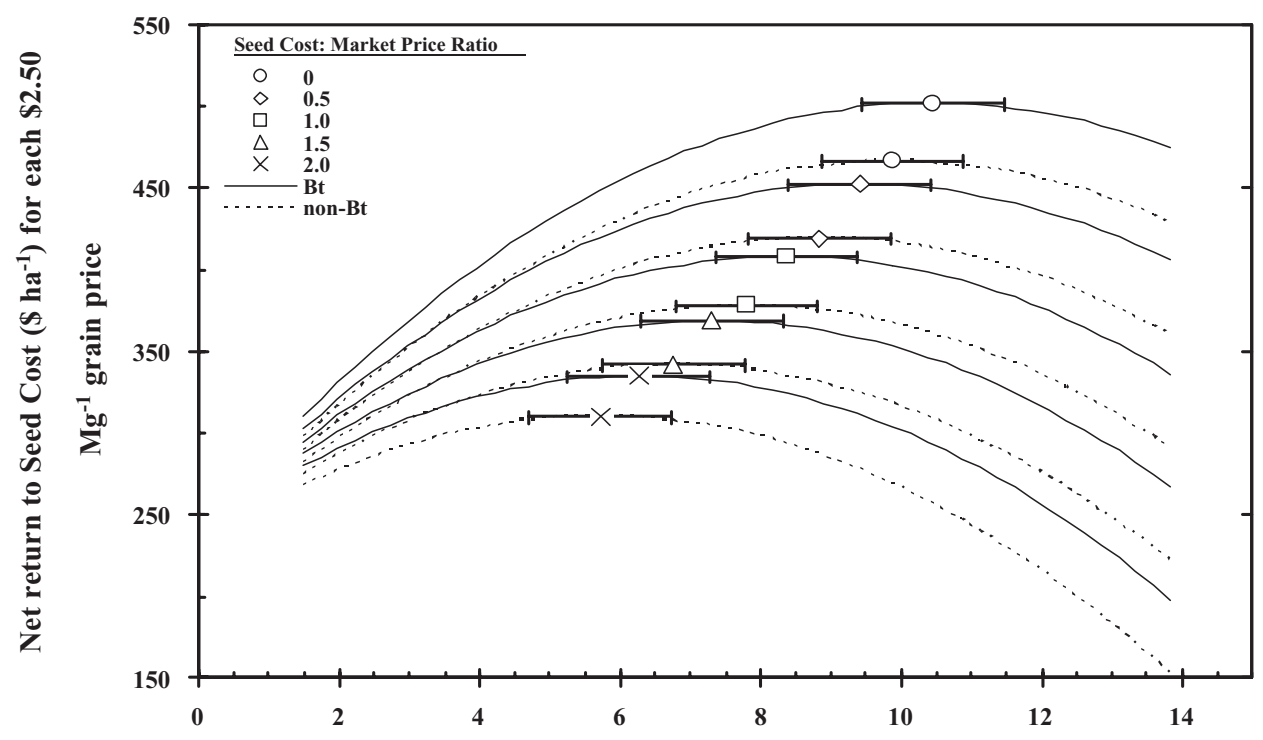

Harvest Population (plants ha ${ }^{-1} x 10$ 000)

Fig. 2. Economic optimum plant population for Bt and non-Bt hybrids for selected seed cost: $\operatorname{market}$ price ratios. Seed costs $=\$ 1000$ seeds ${ }^{-1}$; Market Price $=\$ \mathrm{Mg}^{-1}$; Error bars $= \pm \$ 2.50 \mathrm{ha}^{-1}$.

seed cost, expected market price, and the risk of lodging. Using the yield of $\mathrm{Bt}$ and non-Bt hybrids from this experiment, an economic model was developed to predict grower returns of $\mathrm{Bt}$ and non-Bt corn hybrids over a range of target populations using different seed costs $\left(\$ 1000\right.$ seeds $\left.^{-1}\right)$ : corn market price $\left(\$ \mathrm{Mg}^{-1}\right)$ ratios (Fig. 2). The plant population recommendations for maximizing return were similar for Bt and non-Bt hybrids. Bt hybrids showed a greater return $(7 \%)$ over non-Bt hybrids in all scenarios due to a greater yield potential. The highest gains for increasing plant population came when seed costs were low and market prices were high.

This model is used only to demonstrate how planting decisions in the spring are affected by seed costs and market prices. Because market prices at planting time in the spring can only be estimated, corn growers should base seed purchases on desired seed traits and then adjust planting populations accordingly to maximize yield and profit.

\section{CONCLUSION}

Regardless of corn hybrid trait, this study identified MYPP to be approximately 102400 plants ha ${ }^{-1}$. Further, it was determined that MYPP for Bt hybrids should be 104500 plants ha $^{-1}$ and 98800 plants ha $^{-1}$ for non-Bt hybrids. If plant populations were increased from 74100 plants ha ${ }^{-1}$ to 102400 plants ha $^{-1}$, yields would increase by $4.2 \%$. However, corn management systems must be justified on the basis of economic returns, rather than on crop yield alone (VanGessel et al., 1995). Overall, Bt corn hybrids yielded $6.6 \%$ greater and had $22 \%$ less lodging than non-Bt hybrids. However, the yield and lodging benefits for Bt hybrids were offset by the higher seed and harvest costs associated with Bt corn, adding no economic benefit. This study determined the EOPP to be 83800 plants ha ${ }^{-1}$ (regardless of hybrid trait) or 9700 plants $\mathrm{ha}^{-1}$ more than the current recommendation in Wisconsin.

Farmers and seed companies should continue to periodically evaluate the plant population response of newly released hybrids in specific growing regions to accurately adjust plant population recommendations. In doing so, it is important to keep in mind that economics plays a key role in these recommendations.

\section{ACKNOWLEDGMENTS}

The authors would like to acknowledge the contribution of research specialists Kent Kohn and Pat Flannery for their technical assistance; Jung Won Mun and Paul Jenkins for their assistance in data analysis; and to the associate editor and reviewers for the helpful comments. Funding for this research and publication was provided from the USDA Cooperative State Research, Education and Extension Service (CSREES) project WIS0 142-4897.

\section{REFERENCES}

Armstrong, C.L., G.B. Parker, J.C. Pershing, S.M. Brown, P.R. Sanders, D.R. Duncan, T. Stone, D.A. Dean, D.L. DeBoer, J. Hart et al. 1995. Field evaluation of European corn borer control of progeny of 173 transgenic corn events expressing an insecticidal protein from Bacillus thuringiensis. Crop Sci. 35:550-557.

Bode, W.M., and D.D. Calvin. 1990. Yield-loss relationships and economic injury levels for European corn borer (Lepidoptera: Crambidae) populations infesting Pennsylvania field corn. J. Econ. Entomol. 83:1595-1603.

Bruns, H.A., and H.K. Abbas. 2005. Ultra-high plant populations and nitrogen fertility effects on corn in the Mississippi valley. Agron. J. 97:1136-1140.

Bruns, H.A., and H.K. Abbas. 2006. Planting date effects on Bt and non-Bt corn in the mid-south USA. Agron. J. 98:100-106.

Carmer, S.G., and J.A. Jackobs. 1965. An exponential model for predicting optimum plant density and maximum corn yield. Agron. J. 57:241-244.

Cox, W.J. 1997. Corn silage and grain yield responses to plant densities. J. Prod. Agric. 10:405-410. 
Crosbie, T.M. 1982. Changes in physiological traits associated with long-term breeding efforts to improve grain yield of maize. p. 206233. In H.D. Loden and D. Wilkinson (ed.) Proc. 37th Annu. Corn and Sorghum Industry. Res. Conf., Chicago, IL. 5-9 Dec. 1982. Am. Seed Trade Assoc., Washington, DC.

Dillehay, B.L., G.W. Roth, D.D. Calvin, R.J. Kratochvil, G.A. Kuldau, and J.A. Hyde. 2004. Performance of Bt corn hybrids, their near isolines, and leading corn hybrids in Pennsylvania and Maryland. Agron. J. 96:818-824.

Duffy, M.D. 2004. Estimated costs of crop production in Iowa. Coop. Ext. Serv., FM 1712. Iowa State Univ., Ames.

Duvick, D.N., and K.G. Cassman. 1999. Post-green revolution trends in yield potential of temperate maize in the north-central United States. Crop Sci. 39:1622-1630.

Graeber, J.V., E.D. Nafziger, and D.W. Mies. 1999. Evaluation of transgenic, Bt-containing corn hybrids. J. Prod. Agric. 12:659-663.

Knapp, W.R., and W.S. Reid. 1981. Interactions of hybrid maturity class, planting date, plant population and nitrogen fertilizer on corn performance in New York. Search Agriculture. Agric. Exp. Stn. Bull. 21, Cornell Univ., Ithaca, NY.

Koziel, M.G., G.L. Beland, C. Bowman, N.B. Carozzi, R. Crenshaw, L. Crossland, J. Dawson, N. Desai, M. Hill, and S. Kadwell. 1993. Field performance of elite transgenic maize plants expressing an insecticidal protein derived from Bacillus thuringiensis. Biotechnology 11:194-200.

Lauer, J.G., P.R. Carter, T.M. Wood, G. Diezel, D.W. Wiersma, R.E. Rand, and M.J. Mlynarek. 1999. Corn hybrid response to planting date in the Northern Corn Belt. Agron. J. 91:834-839.

Lauer, J.G., and J. Wedberg. 1999. Grain yield of initial Bt corn hybrid introductions to farmers in the northern corn belt. J. Prod. Agric. 12:373-376.

Littell, R.C., G.A. Milliken, W.W. Stroup, and W.W. Wolfinger. 1996. SAS system for mixed models. SAS Institute, Cary, NC.
Nafziger, E. 1994. Corn planting date and plant population. J. Prod. Agric. 7:59-62.

Olson, R.A., and D.H. Sander. 1988. Corn production. p. 639-686. In G.F. Sprague and J.W. Dudley (ed.) Corn and corn improvement ASA, CSSA, and SSSA, Madison, WI.

Pedersen, P., and J.G. Lauer. 2002. Influence of rotation sequence on the optimum corn and soybean plant population. Agron. J. 94: 968-974.

Porter, P.M., D.R. Hicks, W.E. Lueschen, J.H. Ford, D.D. Warnes, and T.R. Hoverstad. 1997. Corn response to row width and plant density in the Northern Corn Belt. J. Prod. Agric. 10:293-300.

Ritchie, S.W., J.J. Hanway, and G.O. Benson. 1993. How a corn plant develops. Rep. 48. Iowa State Coop. Ext., Iowa State Univ., Ames.

SAS Institute. 2001. SAS release 8.02. SAS Inst., Cary, NC.

Seydou, B.T., R.E. Carlson, C.D. Pilcher, and M.E. Rice. 2000. Bt and non-Bt maize growth and development as affected by temperature and drought stress. Agron. J. 92:1027-1035.

Sibale, E.M., L.L. Darrah, and M.S. Zuber. 1992. Comparison of two rind penetrometers for measurement of stalk strength in maize. Maydica 37:111-114.

Singer, J.W., R.W. Taylor, and W.J. Bamka. 2003. Corn yield response of Bt and near-isolines to plant density. Available at www. plantmanagementnetwork.org/cm/. Crop Manage. doi:10.1094/CM2003-0829-01-RS

Stringfield, G.H., and L.E. Thatcher. 1947. Stands and methods of planting corn hybrids. Agron. J. 39:995-1010.

Swinton, S.M., and J. Lowenberg-DeBoer. 1998. Evaluating the profitability of site-specific farming. J. Prod. Agric. 11:439-446.

VanGessel, M.J., E.E. Schweizer, D.W. Lybecker, and P. Westra. 1995. Compatibility and efficiency of in-row cultivation for weed management in corn. Weed Technol. 9:754-760.

Widdicombe, W.D., and K.D. Thelen. 2002. Row width and plant density effects on corn grain production in the Northern Corn Belt. Agron. J. 94:1020-1023. 